\title{
Fear in Multiple Sclerosis
}

ISSN: 2637-7934

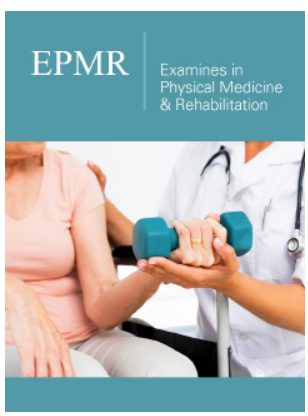

*Corresponding author: Any Docu A, Department of General Medicine, Romania

Submission: 侮 May 29, 2019

Published: 海June 06, 2019

Volume 2 - Issue 4

How to cite this article: Any Docu A, Daniel DA. Fear in Multiple Sclerosis. Examines Phy Med Rehab.2(4). EPMR.000542.2019. DOI: 10.31031/EPMR.2019.02.000542.

Copyright@ Any Docu A, This article is distributed under the terms of the Creative Commons Attribution 4.0 International License, which permits unrestricted use and redistribution provided that the original author and source are credited.

\author{
Any Docu $\mathrm{A}^{1 *}$ and Daniel $\mathrm{DA}^{2}$ \\ ${ }^{1}$ Department of General Medicine, Romania \\ ${ }^{2}$ Department of Physical Education and Sport, Romania
}

\begin{abstract}
The purpose of this study was to identify the connection between fear and multiple sclerosis, due to the fact that numerous patients with this disease have anxiety and are so fearful to even live their life. The fear is related to so many reasons that we commence to analyze it, being attracted by the numerous fields that can connect the disease and this symptom, although, fear is not classified as a manifestation of multiple sclerosis yet. The. fear of the future was found to be a preponderant concern amongst the participants. Affiliated with this fear, the patients evoked peculiar concerns about experiencing farther losses of mobility and independence, becoming a burden on family and caregivers, and having to move to a nursing home.
\end{abstract}

Keywords: Multiple sclerosis; Fear; Anxiety

\section{Introduction}

Multiple sclerosis (MS) is a chronic demyelinating disease and it involves an immunemediated process, in which the immune system responds abnormally, being directed against the central nervous system (brain, spinal cord and optic nerves). The demyelinating process aims the myelin that is located on the neurons of the central nervous system. The role of the myelin is to promote an effective transmission of the impulse of the nerve along the axon and it is produced by oligodendrocytes-another aim of the MS. Likewise, defective immune system response produces inflammation in the central nervous system, affecting: the underlying nerve fiber (lessons or scars that can be detected on the magnetic resonance imaging-MRI) and in this way, it slows the nerve conduction, producing the neurologic signs and symptoms.

The symptomatology in multiple sclerosis is part of a wide range seeking: cranial nerve dysfunction, impairment of ocular motor pathways, impairment of facial sensation, sensory pathways disfunction, motor pathways disfunction, cerebellar pathways disfunction or even affective disorders and cognitive impairment. Fear is an emotional response induced by a distinguished danger or threat, which determines a change in the metabolism and in organ function and ultimately affects the behavior of humans. Fear for people may appear in response to certain stimuli that are currently occurring or as expectant or anticipated risks to life or body of the future. In the face of imminent danger, the man has two options: to run or to fight.

Fear is an emotion that is essential for the organization of defensive behaviors to danger, and therefore has an important role in survival.

The neuro-anatomic system that produces and controls the feeling of fear is complex, consisting of the temporal lobe, the amygdala and the hippocampus. The amygdala is composed of several nuclei that are vital to the acquisition and to the stocking of fear memories. Fear is omnipresent in people's everyday lives and even if it appears for even a few moments, it is a useful tool because its important role can save lives in some cases. The misuse and the attribution in excess of fear leads to pathogenic conditions anxiety or phobia syndromes.

\section{Objective}

This study aims to investigate the fear in multiple sclerosis and the fear in healthy control group and how it reacts in time, while using combined relaxing techniques. 


\section{Method}

A lot of 5 patients with recurrent-remissive multiple sclerosis was recruited from a private clinic. Patients were individually diagnosed by doctor Docu Axelerad Any, the director of the clinic and all met the standard criteria for multiple sclerosis. The control group was composed by 5 healthy patients with mild anxiety disorders. The two groups were advisedly elected to not differ significantly in terms of age:
A. SM range: $20-40$ years
B. SM mean: 28,2 years
C. Control group range: $20-40$ years
D. Control group mean: 30,3 years

\section{Result}

Refined the Expanded Disability Status Scale (EDSS), a useful tool for the quantification of the disability in multiple sclerosis, and we used this scale to determine our patients' condition of being unable to perform as a consequence of physical or mental unfitness, before the beginning of the relaxing techniques [1]. The results were satisfying as following: two patients have an EDSS score of 1 point, two patients have 2 points on EDSS scale and only one patient was framed for 3 points score on the EDSS test 1 . We performed The Hamilton Anxiety Scale (HAS) 2 on our participants before the beginning of the relaxing techniques: both SM patients and control group. The HAS is the one of the first anxiety rating scales measuring the severity of the anxiety symptoms. The results were: the SM patients obtained results in the range of 18-24, framing for mild to moderate severity of anxiety and the control group obtained results in the range of: $<17$ and were included in: mild severity anxiety.

Also, we performed the Hospital Anxiety and Depression Scale (HAD) 3 before the beginning of the relaxing techniques, used to determine the levels of anxiety and depression that a person is experiencing. The results were the following: for the SM patients: the depression score was between 11-21 points-being abnormal and the anxiety score was between $11-21$ points-abnormal. The results for the control group were: the depression scale was between 0-7 points-being normal and the anxiety score was between: 8-10 points-being borderline abnormal.

Interviewing our MS patients, we tried to understand their feelings, emotions and everyday life because having a long-term condition such as MS can be challenging. Confronting with daily unpredictability, along with ample uncertainty about the future, can surely influence one's emotions, feeling frightened or angry, sad, anxious, guilty, worried-these all being predictable reactions to dealing with any chronic condition that may come and go at various points. MS can make patients feel emotional not for a single cause, but in the opposite: because of several reasons.

While most of their feelings is probably due to a reaction to the challenges of living with MS, the emotional changing states can also represent symptoms of the condition which may came as a result of nerve damage to certain areas of the brain. Also, side effects of some drugs (steroids-can cause anxiety, interferon betas-might cause depression) used in the treatment of MS or used in the treatment of MS' symptoms can also cause emotional swings.

We understood from our patients that the fear intricating multiple sclerosis begins to be present in one's life even before the diagnostic's confirmation because even being suspect of such a debilitating disease raises fears and dark scenarios for every human being, having a huge emotional impact. After being confirmed, the disease changes one's life. With the patients' consents we made a few summaries of the cases of each patient with multiple sclerosis, integrating the deficits experienced in the illness and the emotions they feel and even the fears they currently have.

The first patient, female, 37 years, with reccurent-remissive MS, currently EDSS score: 3-mild-moderate hemiparesis, was diagnosed 8 years ago, after taking one year to be diagnosed. She remembers that period being the most difficult one in her life, living in fear every single day and not being able to sleep well at night. She experienced: daily panic attacks with the imminent death sensation, she felt the fear of losing her husband, the fear of losing her job and all her universe was closed and darken up by the fear of having MS [2]. She remembers having tachycardia, a distal tremor, the choking sensation, amongst headache and epigastric pain. In present, the panic attacks happen only once or twice a week, the fear of losing her husband is still present because of her denial to express her feelings and the refusal to talk openly to him.

She is also frightened about the fact that if her disease will accentuate, becoming tetraplegic and her husband will leave her, she will end up in an institution and the three motives she fears being there are: firstly because she doesn't possess the money for the spending there, secondly because of the environment thererepresented mainly by dementia patients and thirdly because she will die there alone. The second patient, male, 28 years, with reccurent-remissive MS, currently EDSS score: 2-mild decrease in the position sense, diagnosed 3 years ago, being in denial a long period of time, now fears of losing the position sense completely and thus falling often and having an instable life physically as well as mentally. His worst fear is about the disease's evolution and is manifested of upsetting restlessness.

The third patient, female, 29 years, with reccurent-remissive MS, currently EDSS score: 2-mild ataxia, diagnosed 4 years ago, has still a hard time accepting the diagnosis, she currently is negatively thinking about the future, having daily visions of herself falling in public places or at home, or being immobilized in a wheelchair, being $100 \%$ dependent of the caregivers. She has chronic insomnia, is over-anticipating facts, is excessively concerned about physical health and is negatively thinking about the future. Her vegetative symptoms are tremor, profuse sweating and suffocation which appear mostly at night [3].

The fourth patient, female, 31 years old, with reccurentremissive MS, currently EDSS score: 1-mild fatigue, has been diagnosed 2 years ago, feels tired all the time and doesn't have energy to do the things that she enjoyed before. Her fear is the 
inability to conceive because of the multiple sclerosis or because of another disease that she might contact meanwhile. She experiences faintness once per day. The fifth patient, female, 35 years, with reccurent-remissive MS, currently EDSS score: 1-mild urinary urgency, has been diagnosed for 6 years, has the constant fear that her children would inherit her disease. She is over-anticipating facts, is excessively concerned about physical health and is negatively thinking about the future (Table $1 \&$ Table 2).

Table 1: In the control group, questionnaire included blank spaces to be completed by every person, with the subject of their fears, we summarized the answers as following.

\begin{tabular}{|c|c|}
\hline Fear of death & $3 / 5$ \\
\hline Fear of getting sick & $2 / 5$ \\
\hline Fear of being alone & $5 / 5$ \\
\hline Fear of the future & $4 / 5$ \\
\hline Fear of impotence & $1 / 5$ \\
\hline Fear of losing the job & $2 / 5$ \\
\hline Fear of poverty & $3 / 5$ \\
\hline Fear of losing family & $5 / 5$ \\
\hline
\end{tabular}

Table 2: In the control group we found the frequency of the following symptoms related to the thoughts of their fear.

\begin{tabular}{|c|c|}
\hline Panic attacks & $2 / 5$ \\
\hline Increased heart rate & $4 / 5$ \\
\hline Palpitations & $2 / 5$ \\
\hline Dry mouth & $2 / 5$ \\
\hline Shortness of breath & $2 / 5$ \\
\hline Nausea & $2 / 5$ \\
\hline Faintness & $2 / 5$ \\
\hline Restlessness & $1 / 5$ \\
\hline Hot or cold sensations & $1 / 5$ \\
\hline Tingling in extremities & $1 / 5$ \\
\hline Muscular tension & $1 / 5$ \\
\hline
\end{tabular}

The multiple sclerosis patients and the control group were advised to practice combined relaxing techniques: meditation and floating (in a pool), both of them once per day for 30minutes, for 6 months. The floating lessons contained distal member movements, as much as possible to the patient. The lessons of meditation consisted more in developing breathing techniques and concentration of thoughts, in externalizing the negative ones and implicitly externalizing the fears. The meditation and swimming instructors remarked an improving evolution for every patient during the six months and especially have observed a relaxation of the mental, which is also reflected in the physical, the patients being less tense and contracted.

We performed The Hamilton Anxiety Scale (HAS) 2 on our participants at the end of the six months practice of the relaxing techniques: both SM patients and control group. The results were: three SM patients obtained results in the range of: 18-24, framing for mild to moderate severity of anxiety and two patients obtained results in the range of $<17$ and were included in: mild severity anxiety. The control group obtained results in the range of: $<17$ and were included in mild severity anxiety, but with the score reduction of minimum two points per patient.

Also, we performed the Hospital Anxiety and Depression Scale (HAD) 3 after the end of the six months practicing relaxing techniques, used to determine the levels of anxiety and depression that a person is experiencing. The results were the following: for the SM patients: the depression score was between: 11-21 points-being abnormal and the anxiety score was between 8-10 points-being borderline abnormal, which is an increase of results of every single patient, that conducted everyone to the superior scale class, from being abnormal to being borderline abnormal related to anxiety scale. The results for the control group were: the depression scale was between 0-7 points-being normal and the anxiety score was between: 8-10 points-being borderline abnormal, the classification was the same, but was observed an evolution for every participant, having scores reduced with minimum one point per patient.

The first patient, female, 37 years, with reccurent-remissive MS, currently EDSS score: 3-mild-moderate hemiparesis, reported an improving in the perception and movement of the affected members. The deficit has decreased in severity (from $3 / 5$ to $4 / 5$ ), tending to become a mild hemiparesis, form a moderate one. She claims that panic attacks have decreased in frequency, now having only one panic attack a week, and sometimes even weeks without panic attacks. After a month of meditation and floating sessions in the pool, she began to have a better relationship with her husband and started to tell him more about how she feels and what is happening to her, and that in the months to come she was accompanied by her husband to the combined relaxation techniques. Now she feels more confident about their relationship and claims to have fewer worrying thoughts.

The second patient, male, 28 years, with reccurent-remissive MS, currently EDSS score: 2-mild decrease in the position sense, claimed to have an improvement in his perception of the position sense, explaining that the floating sessions helped him with a better understanding of his body parts location and the slow movements in the water helped him feel the exact demarcation of his body extremities; and about the meditation sessions he claimed that by meditating he obtained a boosted self-confidence and a peace of mind that helped him to worry less about the future in terms of a debilitating future. The restlessness became mild and only in moments of stress.

The third patient, female, 29 years, with reccurent-remissive MS, currently EDSS score: 2-mild ataxia. the patient concluded that combined relaxation therapies helped her not to fear the idea of falling, and also helped her to gain greater confidence in her, but the ataxia was still present, the patient quantifying a reduction in ataxia frequency by up to $20 \%$. We advised her to monitor her insomnia and we observed a progress: in every month she had less nights with insomnia that in previous month. She explained that having activity both mental and physical, helped her with the over thinking and with the over-anticipating facts, having a decreasing 
frequency of worrying. She related that she feels healthier or she is concerning less frequent about diseases she may have or contact.

The fourth patient, female, 31 years old, with reccurentremissive MS, currently EDSS score: 1-mild fatigue, relates that she feels more energic and that the severity of the fatigue has decreased, she is becoming tired only by the afternoon. She started with floating technique once per day for 30 minutes and she increased the time in the pool and the exercises so at the end of the 6 months, she had sessions of 60 minutes in the water, swimming and making special exercises for the muscle tonus, due this fact she felt stronger both physical and mental and had appointments with an expert advising on couple fertility issues and she and her husband had meetings with another couple with a child born from a mother with multiple sclerosis.

For the fifth patient, female, 35 years, with reccurent-remissive MS, currently EDSS score: 1-mild urinary urgency, the combined relaxing techniques were done initially with breaks at 5-10 minutes, due to the fear of the patient that had the sensation of urinary urgency often, so the therapist and the instructors understood her and gave her the freedom of having frequent breaks at 5-10 minutes for how long time she would want. After three months, she had only one or two interruptions and in the final month she experienced sessions with no breaks. She explained that she obtained selfconfidence and got to know herself better.

Table 3: At the end of the six months, the same parameters were analized at every patient, with the following conclusions.

\begin{tabular}{|c|c|}
\hline Fear of death & $1 / 5$ \\
\hline Fear of getting sick & $1 / 5$ \\
\hline Fear of being alone & $2 / 5$ \\
\hline Fear of the future & $3 / 5$ \\
\hline Fear of impotence & $1 / 5$ \\
\hline Fear of losing the job & $2 / 5$ \\
\hline Fear of poverty & $2 / 5$ \\
\hline Fear of losing family & $3 / 5$ \\
\hline
\end{tabular}

In the control group the combined relaxing methods were very popular and beneficial for the patient's mental, so every single one patient requested the permission to make two sessions of both meditation and floating per day. Their requirement was welcomed by the team, after analyzing their improvement after a month (Table 3 ). The fear of death decreased in the study control group because of the gaining of self-confidence, related the participants, due to the fact that they felt stronger and healthier (also the fear of getting sick decreased). Three patients were not afraid to remain alone due to the fact that they observed that loneliness is not so unbearable that they thought. Having meaningful and useful activities for both body and mind, helped them to feel more self-conscious. The fear of losing the family was defeated by the fact that two patients engaged their closest family members in their activities and even practiced together, even that not always in the same time. Because of decreasing the magnitude of those fears, the participants started to visualize a brighter future, with less fear and over-anticipating facts (Table 4).

Table 4: In the control group we quantified the frequency of the symptoms related to the thoughts of their fear after the six months of combined relaxing techniques and obtained the following data.

\begin{tabular}{|c|c|}
\hline Panic attacks & $0 / 5$ \\
\hline Increased heart rate & $2 / 5$ \\
\hline Palpitations & $0 / 5$ \\
\hline Dry mouth & $2 / 5$ \\
\hline Shortness of breath & $2 / 5$ \\
\hline Nausea & $2 / 5$ \\
\hline Faintness & $0 / 5$ \\
\hline Restlessness & $1 / 5$ \\
\hline Hot or cold sensations & $1 / 5$ \\
\hline Tingling in extremities & $1 / 5$ \\
\hline Muscular tension & $1 / 5$ \\
\hline
\end{tabular}

The panic attacks, palpitations and faintness were not encountered between our participants of the controlgroup at the end of the combined relaxing techniques, but the vegetative symptoms such as: dry mouth, increased heart rate, shortness of breath and nausea were still present at the same patients, though they claimed a less often manifestation of the symptoms. They all concluded that the non-medicinal therapy of relaxing techniques had a beneficial effect on their both physical and mental manifestations of fear. Every single one reported a decreased frequency of the fearful thoughts and a decreased gravity of the thoughts itself. We have to mention that the benefits we observed should be considered with caution and included in rigorous experimental research in the future related to the relaxing combined techniques in fearful patients with reccurent-remissive MS.

\section{References}

1. Kurtzke JF (1983) Rating neurologic impairment in multiple sclerosis: An expanded disability status scale (EDSS). Neurology 33(11): 14441452.

2. Hamilton M (1959) The assessment of anxiety states by rating. Br J Med Psychol 32(1): 50-55.

3. Zigmond AS, Snaith RP (1983) The hospital anxiety and depression scale. Acta Psychiatrica Scandinavica 67(6): 361-370. 